\title{
ÖFFENTLICHE ENTWICKLUNGSZUSAMMENARBEIT
}

Die Förderung der wirtschaftlichen und sozialen Entwicklung in Nichtmitgliedsländern ist seit ihrer Gründung eines der Hauptziele der OECD. Der für die öffentliche Entwicklungszusammenarbeit (ODA) bereitgestellte Anteil des Nationaleinkommens ist ein Prüfstein für das Engagement eines Landes zu Gunsten der internationalen Entwicklung. Ein bereits vor langer Zeit festgelegtes Ziel der Vereinten Nationen ist, dass die Industriestaaten 0,7\% ihres Bruttonationaleinkommens (BNE) als ODA zur Verfügung stellen sollten.

\section{Definition}

Die ODA ist definiert als staatliche Unterstützung, die das Ziel verfolgt, die wirtschaftliche Entwicklung und das Wohlergehen der Entwicklungsländer zu fördern. Darlehen und Kredite für militärische Zwecke sind nicht einbezogen. Die Leistungen der Entwicklungszusammenarbeit (EZ) können den Empfängern entweder bilateral von den Gebern oder über eine multilaterale Entwicklungsorganisation, wie die Vereinten Nationen oder die Weltbank, zur Verfügung gestellt werden. Die ODA umfasst Zuschüsse, „weiche“ Kredite sowie technische Zusammenarbeit. Bei weichen Krediten beträgt das Zuschusselement mindestens $25 \%$ des Gesamtbetrags.

Die OECD hat eine Liste von Entwicklungsländern und -gebieten aufgestellt; lediglich die EZ-Leistungen an diese Länder werden als ODA gezählt. Die Liste wird regelmäßig aktualisiert und umfasst derzeit mehr als 150 Länder oder Gebiete, deren

\section{Überblick}

Zwischen 1960 und 1990 stiegen die ODA-Leistungen der DAC-Mitgliedsländer an die Entwicklungsländer stetig an. Die gesamten ODA-Leistungen in Prozent des kombinierten BNE der DAC-Mitgliedsländer sanken dagegen zwischen 1960 und 1970 und schwankten danach über einen Zeitraum von etwas mehr als zwanzig Jahren zwischen 0,27\% und 0,36\%. Zwischen 1993 und 1997 gingen die ODALeistungen auf Grund der Haushaltskonsolidierung in den Geberländern im Gefolge der Rezession Anfang der 1990er Jahre in realer Rechnung um $16 \%$ zurück.

Ab 1998 nahmen die Leistungen in realer Rechnung dann wieder $\mathrm{zu}$, und seither wurden die ODA-Leistungen auf einer Reihe internationaler Konferenzen, die viel Aufmerksamkeit auf sich gezogen haben, aufgestockt. 2002 wurden auf der Internationalen Konferenz über Entwicklungsfinanzierung in Monterrey, Mexiko, für jeden Geber feste Ziele aufgestellt, und in der Folge zeigten die ODALeistungen nach einem Jahrzehnt des Rückgangs wieder eine Aufwärtstendenz. 2005 gingen die Geber auf dem G8Gipfel in Gleneagles und beim VN-Millennium+5-Gipfel weitere Verpflichtungen zur Erhöhung der ODA-Leistungen ein.

Die Netto-ODA-Leistungen stiegen zwischen 2000 und 2010, dem Jahr, in dem der Höchststand erreicht wurde, um 63\%. 2011 sanken die gesamten ODA-Nettoleistungen der DAC-Mitglieder auf 133,5 Mrd. US-\$, was gegenüber 2010 einem Rückgang um 2,7\% in realer Rechnung entspricht. Der gewichtete Durchschnitt der gesamten ODA-Leistungen in Prozent des kombinierten Geber-BNE lag 2011 bei $0,31 \%$.
Pro-Kopf-Einkommen 2010 unter 12276 US-\$ lag. Die Daten über die ODA-Leistungen werden von den 24 OECD-Ländern bereitgestellt, die Mitglieder des Entwicklungsausschusses (DAC) sind.

\section{Vergleichbarkeit}

Die ODA-Statistiken werden nach vom DAC erarbeiteten Leitlinien erstellt. Die Statistiken für die einzelnen Länder werden regelmäßig im Rahmen von Peer Reviews durch andere DACMitgliedsländer geprüft.

\section{ODA-Nettoleistungen} 1960-2011

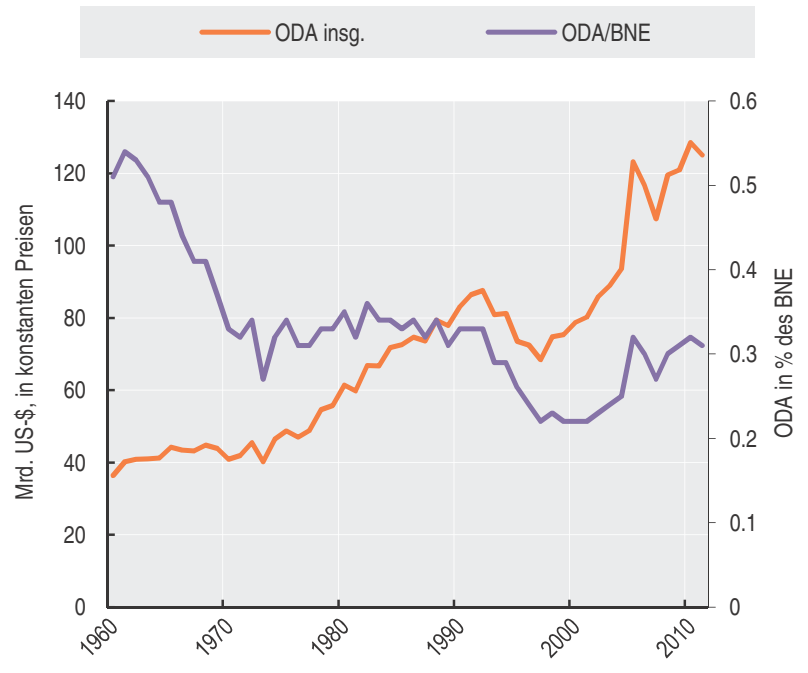

StatLink 前S $h t t p: / / d x . d o i . o r g / 10.1787 / 888932710270$

\section{Quelle}

- OECD (2011), OECD International Development Statistics, OECD Publishing.

\section{Weitere Informationen}

\section{Analysen}

- Keeley, B. (2012), From Aid to Development, The Global Fight against Poverty, OECD Insights, OECD Publishing.

- OECD (2012), Aid Effectiveness 2011, Progress in Implementing the Paris Declaration, Better Aid, OECD Publishing.

- OECD (2010), OECD Journal on Development, OECD Publishing.

Statistiken

- OECD (2011), Geographical Distribution of Financial Flows to Developing Countries, OECD Publishing.

- OECD (2010), Creditor Reporting System, OECD Publishing.

- OECD und Welthandelsorganisation (2011), Aid for Trade at a Glance, OECD Publishing.

Online-Datenbanken

- OECD International Development Statistics.

Websites

- Aid at a glance: by donor, recipient and region, www.oecd.org/ dac/stats/aidcharts.

- OECD Aid Statistics, www.oecd.org/dac/stats. 
ODA-Nettoleistungen

\begin{tabular}{|c|c|c|c|c|c|c|c|c|c|c|c|c|}
\hline & \multicolumn{6}{|c|}{ In Prozent des Bruttonationaleinkommens } & \multicolumn{6}{|c|}{ Mio. US-Dollar } \\
\hline & 2006 & 2007 & 2008 & 2009 & 2010 & 2011 & 2006 & 2007 & 2008 & 2009 & 2010 & 2011 \\
\hline Australien & 0.30 & 0.32 & 0.32 & 0.29 & 0.32 & 0.35 & 2123 & 2669 & 2954 & 2762 & 3826 & 4799 \\
\hline Belgien & 0.50 & 0.43 & 0.48 & 0.55 & 0.64 & 0.53 & 1977 & 1951 & 2386 & 2610 & 3004 & 2800 \\
\hline Dänemark & 0.80 & 0.81 & 0.82 & 0.88 & 0.91 & 0.86 & 2236 & 2562 & 2803 & 2810 & 2871 & 2981 \\
\hline Deutschland & 0.36 & 0.37 & 0.38 & 0.35 & 0.39 & 0.40 & 10435 & 12291 & 13981 & 12079 & 12985 & 14533 \\
\hline Finnland & 0.40 & 0.39 & 0.44 & 0.54 & 0.55 & 0.52 & 834 & 981 & 1166 & 1290 & 1333 & 1409 \\
\hline Frankreich & 0.47 & 0.38 & 0.39 & 0.47 & 0.50 & 0.46 & 10601 & 9884 & 10908 & 12602 & 12915 & 12994 \\
\hline Griechenland & 0.17 & 0.16 & 0.21 & 0.19 & 0.17 & 0.11 & 424 & 501 & 703 & 607 & 508 & 331 \\
\hline Irland & 0.54 & 0.55 & 0.59 & 0.54 & 0.52 & 0.52 & 1022 & 1192 & 1328 & 1006 & 895 & 904 \\
\hline Italien & 0.20 & 0.19 & 0.22 & 0.16 & 0.15 & 0.19 & 3641 & 3971 & 4861 & 3297 & 2996 & 4241 \\
\hline Japan & 0.25 & 0.17 & 0.19 & 0.18 & 0.20 & 0.18 & 11136 & 7697 & 9601 & 9457 & 11021 & 10604 \\
\hline Kanada & 0.29 & 0.29 & 0.33 & 0.30 & 0.34 & 0.31 & 3683 & 4080 & 4795 & 4000 & 5209 & 5291 \\
\hline Korea & 0.05 & 0.07 & 0.09 & 0.10 & 0.12 & 0.12 & 455 & 696 & 802 & 816 & 1174 & 1321 \\
\hline Luxemburg & 0.89 & 0.92 & 0.97 & 1.04 & 1.05 & 0.99 & 291 & 376 & 415 & 415 & 403 & 413 \\
\hline Neuseeland & 0.27 & 0.27 & 0.30 & 0.28 & 0.26 & 0.28 & 259 & 320 & 348 & 309 & 342 & 429 \\
\hline Niederlande & 0.81 & 0.81 & 0.80 & 0.82 & 0.81 & 0.75 & 5452 & 6224 & 6993 & 6426 & 6357 & 6324 \\
\hline Norwegen & 0.89 & 0.95 & 0.89 & 1.06 & 1.10 & 1.00 & 2945 & 3735 & 4006 & 4081 & 4580 & 4936 \\
\hline Österreich & 0.47 & 0.50 & 0.43 & 0.30 & 0.32 & 0.27 & 1498 & 1808 & 1714 & 1142 & 1208 & 1107 \\
\hline Portugal & 0.21 & 0.22 & 0.27 & 0.23 & 0.29 & 0.29 & 396 & 471 & 620 & 513 & 649 & 669 \\
\hline Schweden & 1.02 & 0.93 & 0.98 & 1.12 & 0.97 & 1.02 & 3955 & 4339 & 4732 & 4548 & 4533 & 5606 \\
\hline Schweiz & 0.39 & 0.38 & 0.44 & 0.45 & 0.40 & 0.46 & 1646 & 1685 & 2038 & 2310 & 2300 & 3086 \\
\hline Spanien & 0.32 & 0.37 & 0.45 & 0.46 & 0.43 & 0.29 & 3814 & 5140 & 6867 & 6584 & 5949 & 4264 \\
\hline Ver. Königreich & 0.51 & 0.36 & 0.43 & 0.51 & 0.57 & 0.56 & 12459 & 9849 & 11500 & 11283 & 13053 & 13739 \\
\hline Ver. Staaten & 0.18 & 0.16 & 0.18 & 0.21 & 0.21 & 0.20 & 23532 & 21787 & 26437 & 28831 & 30353 & 30745 \\
\hline
\end{tabular}

StatLink aiाsस $h t t p: / / d x . d o i . o r g / 10.1787 / 888932710232$

\section{Verteilung der ODA-Nettoleistungen aus allen Quellen nach Einkommensgruppe und Regionen}

\begin{tabular}{|c|c|c|c|c|c|}
\hline & 2006 & 2007 & 2008 & 2009 & 2010 \\
\hline \multicolumn{6}{|l|}{ Nach Einkommensgruppe } \\
\hline Am wenigsten entwickelte Länder & 28931 & 34034 & 39162 & 40334 & 44805 \\
\hline Sonstige Länder der unteren Einkommensgruppe & 19203 & 10969 & 10647 & 16042 & 14552 \\
\hline Länder der mittleren Einkommensgruppe, unterer Bereich & 28893 & 29288 & 32570 & 26357 & 25176 \\
\hline Länder der mittleren Einkommensgruppe, oberer Bereich & 6274 & 6405 & 8536 & 7554 & 7374 \\
\hline Nicht zugeordnet & 24014 & 27914 & 37002 & 36681 & 39201 \\
\hline Fortgeschrittene Entwicklungsländer und -gebiete & 24 & -116 & .. &.. & .. \\
\hline \multicolumn{6}{|l|}{ Nach Region } \\
\hline Subsahara-Afrika & 40869 & 34727 & 39627 & 42465 & 43805 \\
\hline Süd- und Zentralasien & 11430 & 14091 & 15981 & 18464 & 18636 \\
\hline Sonstiges Asien und Ozeanien & 8646 & 9585 & 9858 & 10886 & 10628 \\
\hline Naher Osten und Nordafrika & 17058 & 17891 & 24138 & 13370 & 12086 \\
\hline Lateinamerika und Karibik & 7340 & 6987 & 9288 & 9022 & 10718 \\
\hline Europa & 5082 & 4337 & 5377 & 5731 & 5856 \\
\hline Unaufgeschlüsselt & 16913 & 20876 & 23647 & 27028 & 29378 \\
\hline Entwicklungsländer insgesamt & 107339 & 108494 & 127916 & 126968 & 131108 \\
\hline
\end{tabular}

\section{ODA-Nettoleistungen}

2011

Verteilung der ODA-Nettoleistungen aus allen Quellen nach Regionen Mio. US-Dollar

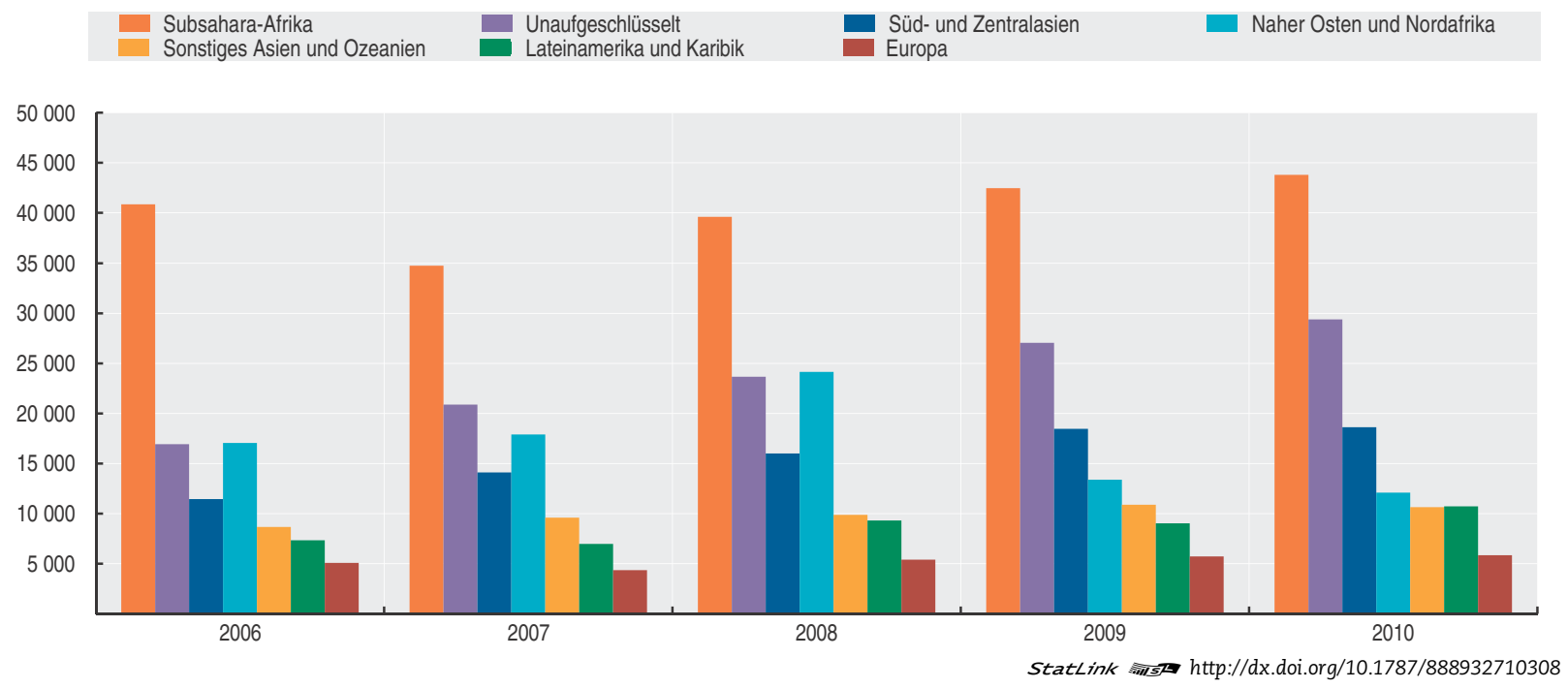

Verteilung der ODA-Nettoleistungen der Länder aus allen Quellen nach Einkommensgruppen Mio. US-Dollar

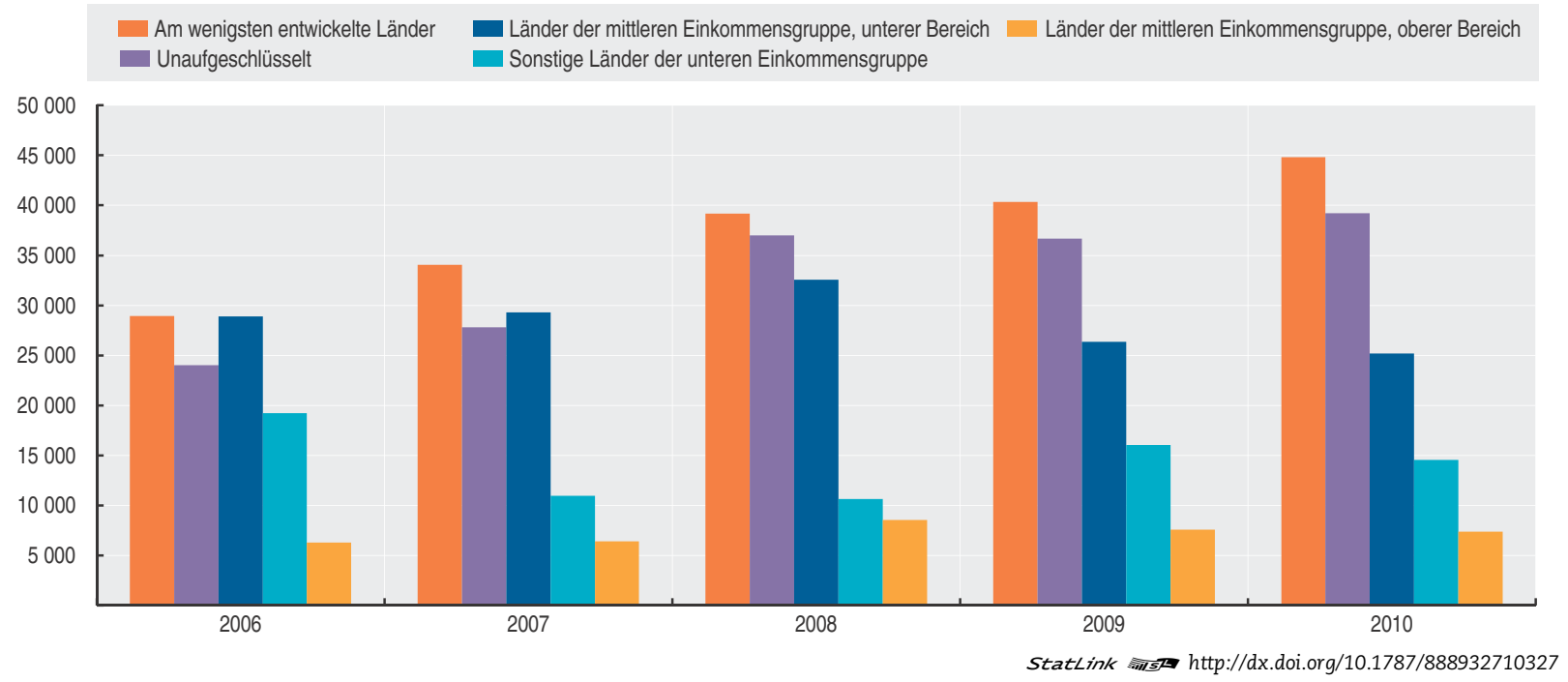

Verteilung der bilateralen ODA-Bruttoleistungen der DAC-Mitglieder nach Einkommensgruppen Mio. US-Dollar, Durchschnitt 2009-2010

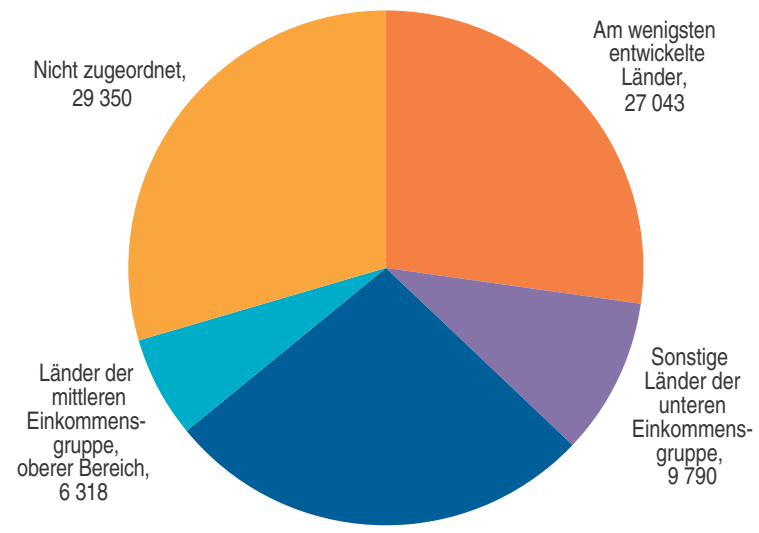

Länder der mittleren Einkommensgruppe, unterer Bereich, 26827

StatLink ails http://dx.doi.org/10.1787/888932710346 


\section{Verteilung der bilateralen ODA-Bruttoleistungen der DAC-Mitglieder nach Regionen}

Mio. US-Dollar, Durchschnitt 2009-2010

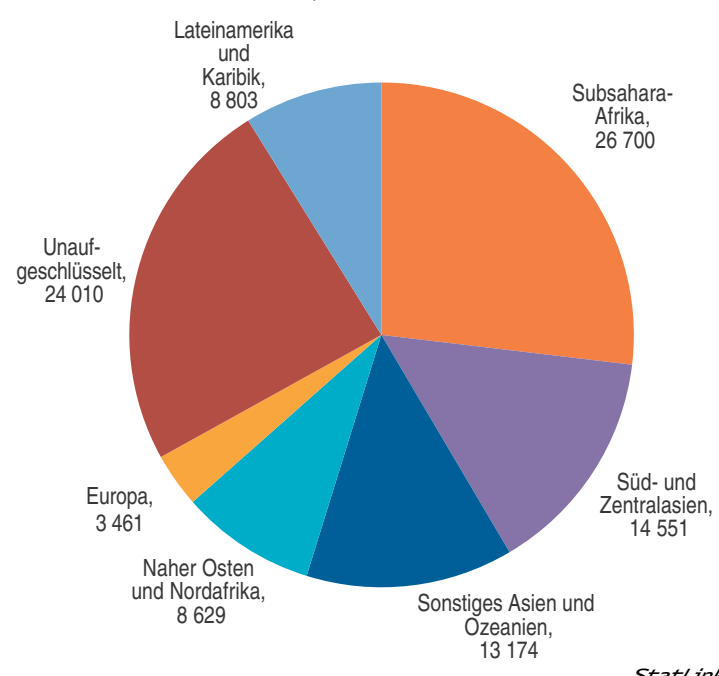

StatLink Aîsस $h t t p: / / d x . d o i . o r g / 10.1787 / 888932710365$

Verteilung der bilateralen ODA-Bruttoleistungen der DAC-Mitglieder nach Sektoren

In Prozent der gesamten bilateralen ODA-Bruttoleistungen, Durchschnitt 2009-2010

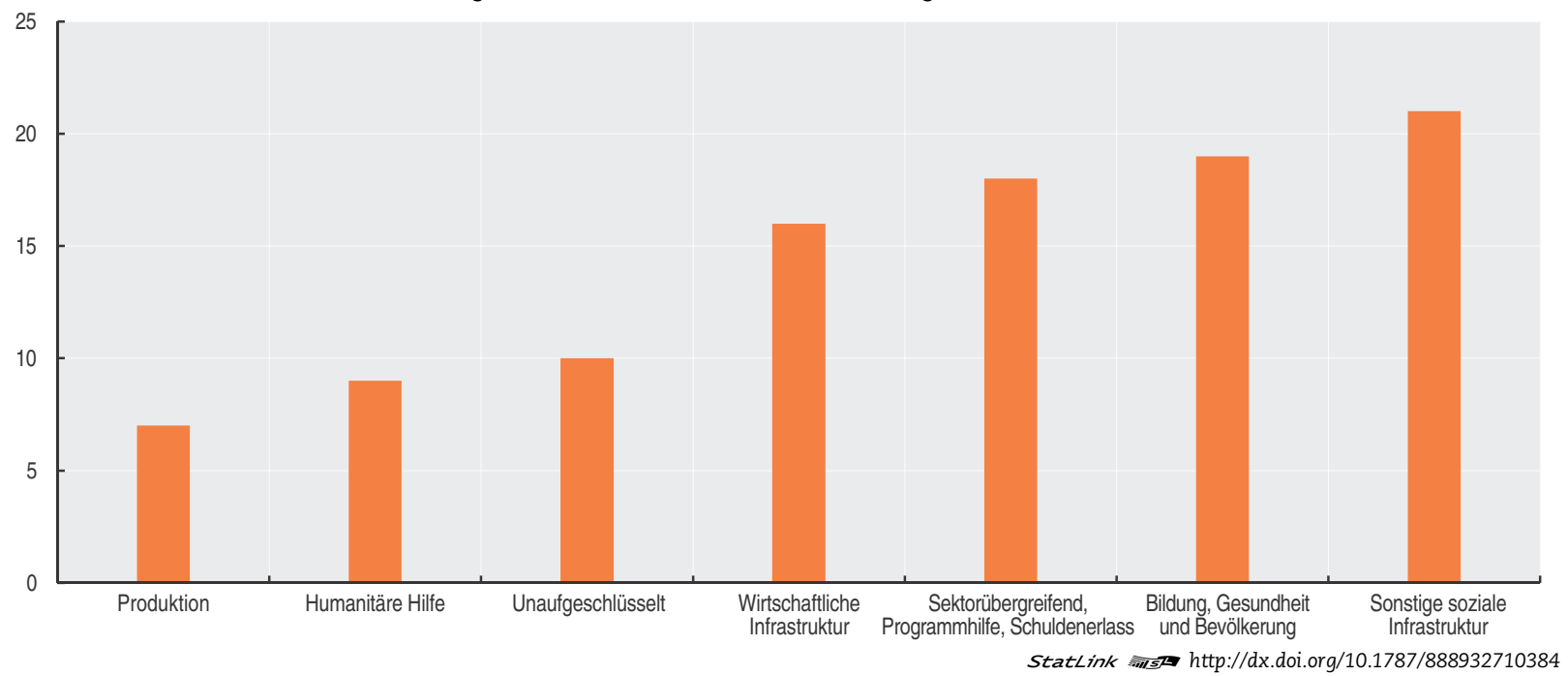

Zusammensetzung der ODA der DAC-Mitgliedsländer

Mio. US-Dollar, 2010

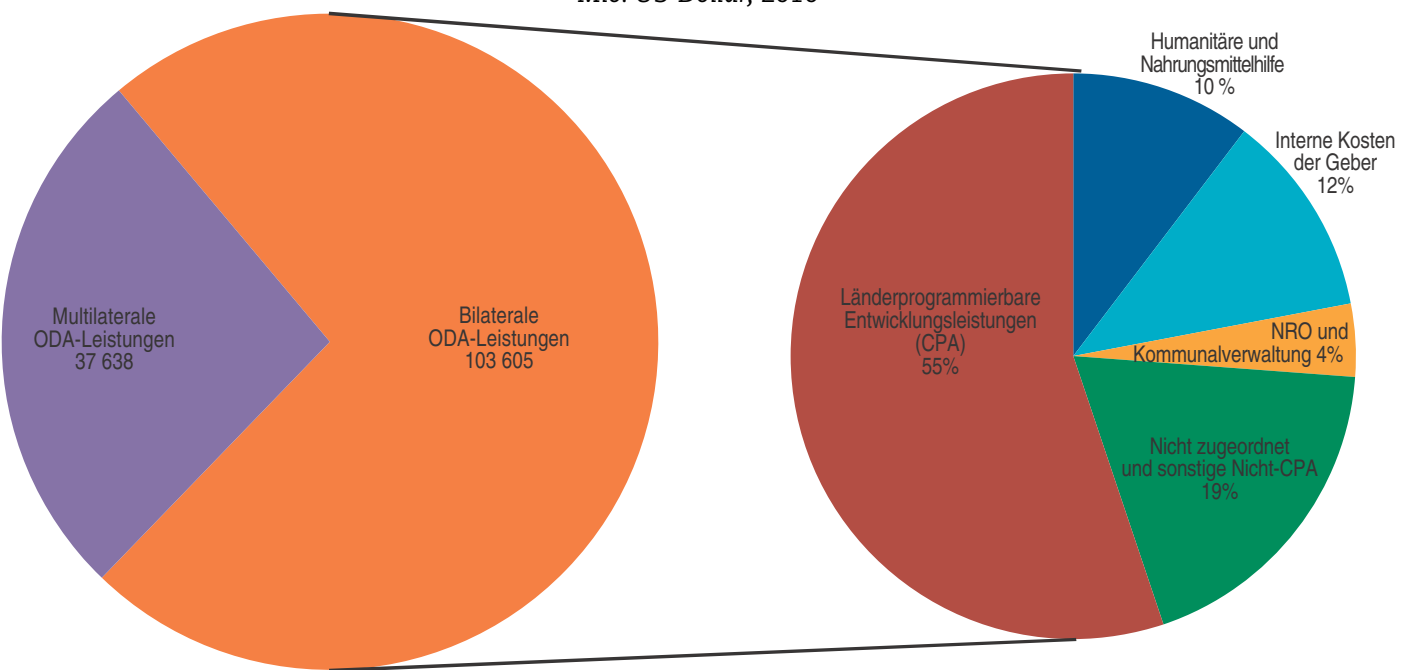

StatLink Ailsय $h t t p: / / d x . d o i . o r g / 10.1787 / 888932710403$ 


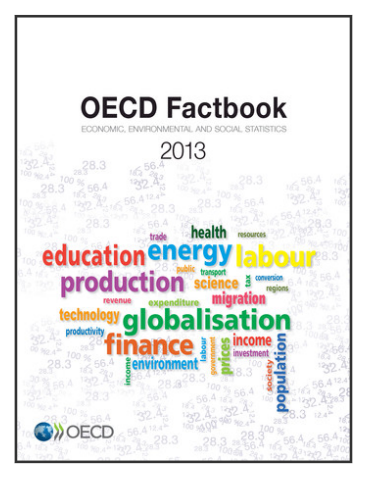

\section{From: \\ OECD Factbook 2013 \\ Economic, Environmental and Social Statistics}

Access the complete publication at:

https://doi.org/10.1787/factbook-2013-en

\section{Please cite this chapter as:}

OECD (2013), "Öffentliche Entwicklungszusammenarbeit”, in OECD Factbook 2013: Economic, Environmental and Social Statistics, OECD Publishing, Paris.

DOI: https://doi.org/10.1787/factbook-2013-92-de

Das vorliegende Dokument wird unter der Verantwortung des Generalsekretärs der OECD veröffentlicht. Die darin zum Ausdruck gebrachten Meinungen und Argumente spiegeln nicht zwangsläufig die offizielle Einstellung der OECDMitgliedstaaten wider.

This document and any map included herein are without prejudice to the status of or sovereignty over any territory, to the delimitation of international frontiers and boundaries and to the name of any territory, city or area.

You can copy, download or print OECD content for your own use, and you can include excerpts from OECD publications, databases and multimedia products in your own documents, presentations, blogs, websites and teaching materials, provided that suitable acknowledgment of OECD as source and copyright owner is given. All requests for public or commercial use and translation rights should be submitted to rights@oecd.org. Requests for permission to photocopy portions of this material for public or commercial use shall be addressed directly to the Copyright Clearance Center (CCC) at info@copyright.com or the Centre français d'exploitation du droit de copie (CFC) at contact@cfcopies.com. 